\title{
Research progress of NPR genes in signal pathway of salicylic acid mediated plant disease resistance
}

\author{
Pan Wang ${ }^{1}$, Meiqin Xiang ${ }^{2}$ \\ ${ }^{1}$ College of Forestry, Beihua University, Jilin 132013, China; \\ ${ }^{2}$ College of Life Science and Technology, Yangtze Normal University, Chongqing 408100, China;
}

\begin{abstract}
Salicylic acid (SA) is considered to be an endogenous signal molecule in plants, and it is related to many resistances in plants. In Arabidopsis, Non-expressor of pathogenesis-related gene1 (NPR1) mediates the expression of pathogenesis-related genes (PRs) and systemic acquired resistance (SAR) induced by SA. NPR1 is a key factor in SA signaling pathway, and the research shows that NPR1, NPR3 and NPR4 play a key role in SA mediated plant disease resistance. In this review, the interaction between NPR and transcription factors is discussed, and we also describe the progress of NPR in SA mediated SAR signal transduction pathway, likewise, we introduce the relationship between NPR1 and its paralogues NPR3/NPR4. This paper analyzes the research prospect of NPR as the intersection of multiple signal paths.
\end{abstract}

\section{Introduction}

Plants are usually infected by a variety of pathogens in the process of growth and development. In order to cope with biological and abiotic stresses in the external environment, plants have gradually formed a series of immune resistance in the long evolution process. Plant immune activities are usually classified as the pattern-triggered immunity (PTI) and the intracellular effector-triggered immunity (ETI). PTI mainly refers to the basic defense response stimulated by pathpgen-asspciated molecular patterns (PAMPs) ${ }^{[1-2]}$. Pathogens avoid the basic defense response of plants by producing effective factors, then plants generate resistance genes to detect the effectors encoded by pathogens and initiate the second level of immune response $\mathrm{ETI}^{[3-4]}$. In general, after ETI is activated by R-gene, the resistance produced by plants has a typical form, the infected site can prevent the spread of pathogens in the way of rapid necrosis of local tissues, which is called hypersensitivity response $(\mathrm{HR})^{[5]}$, and then trigger the corresponding $\mathrm{SAR}^{[6]}$. In addition, the plant defense system also includes the induced systemic resistance (ISR), which refers to the systemic resistance of the whole plant caused by the roots of some nonpathogenic bacteria ${ }^{[7]}$. The accumulation of SA induces the production of SAR, and JA and ET are the key signal molecules to induce the production of ISR, however, the signal transduction pathways mediated by these hormones cross, some inhibit and some promote each other ${ }^{[8]}$.

SA is a simple phenolic compound, which was first found in the extract of willow bark ${ }^{[9]}$.SA can regulate the growth and development of plants and some stress responses, such as inducing flowering, affecting gender differentiation, and regulating plant photoperiod ${ }^{[10-11]}$. SA can induce Arabidopsis, tobacco, corn and other plants to produce resistance, which is considered as a chemical inducer of $\mathrm{SAR}^{[12]}$. The accumulation of exogenous SA is enough to induce the establishment of SAR. Blocking the synthesis or accumulation of SA will lead to the inability of plants to produce SAR, and it is more sensitive to pathogens ${ }^{[13]}$. As a key factor of disease resistance in the process of plant SA signal transduction, course related Non-expressor of pathogenesis-related gene1 NPR1 (also known as NIM1) is located in the upstream of course related factor PR protein gene, which plays a key role in regulating SAR and ISR, and is the intersection of multiple disease resistance signal transduction pathways. In the NPR1 mutant, the gene encoding PR protein could not be expressed, SAR could not be activated, and disease resistance could not be produced, which indicated that the lack of NPR1 would lead to the loss of SAR ${ }^{[14-15]}$. In Arabidopsis, carrot, rice, tobacco, tomato, wheat, apple and many other plants, it was found that the overexpression of NPR1 gene would lead to the improvement of plant disease resistance ${ }^{[16-19]}$.

When the plant is infected by pathogenic bacteria, the accumulation of SA in the plant induces the monomer of NPR1 by destroying the disulfide bond between cysteine through Trxs, and causes the change of redox state in the cell, thus affecting the NPR1 signaling pathway ${ }^{[20]}$.The research shows that there are five amino acid sequences similar to NPR1 in Arabidopsis gene tissue, which are named npr2, npr3, npr4 npr5 and npr6, in addition, the research of the function of npr4 showed that the gene was resistant to Pseudomonas syringae and Erysiphe cichoracearum $^{[21]}$. On the contrary, npr3 and npr4, the nprl-like genes in Arabidopsis, showed negative regulation on PR gene expression in Arabidopsis ${ }^{[22]}$. In this review, we first introduce the interaction mechanism 
between NPR1 and transcription factors. And we then summarize and analyze the relationship between members of NPR gene family and the mechanism of interaction and response to SA mediated plant disease resistance. Altogether, we discuss the mechanism of NPR in plant disease resistance induced by SA, and the plant signal defense mechanism mediated by SA is a precise and complex process.

\section{The mechanism of NPR in plant disease resistance}

The research shows that although the SA accumulated by Arabidopsis NPR1 mutants under SAR induction can reach the normal level, it cannot be excited in Arabidopsis and rice, overexpression of NPR1 can improve the plant's resistance to pathogens and fungal viruses, which indicates that NPR1 is a key regulator downstream of SA in SAR signal transduction process ${ }^{[23]}$. It can be seen that NPR1 gene can regulate the occurrence of SAR in plants: in the absence of NPR1, Arabidopsis mutant plants are not only unable to respond to various SAR inducers, but also have little expression of PR gene. The symptoms of infection by pathogenic microorganisms are obvious, that is, the sensitivity is increased ${ }^{[13]}$. On the contrary, in the SA signal transduction pathway of Arabidopsis, NPR1 gene is different when it exists, which can induce SAR process to produce key regulatory components and participate in the activation of PR gene expression. In addition, the level of SA in uninfected tissues increased after infection.

\section{1 the interaction between NPR and transcription factors}

NPR1 exists in the form of complex oligomer, and its reduction potential increases when it is induced by SA, the disulfide bond between 82 and 216 cysteine residues connecting NPR1 oligomer is reduced and broken, and NPR1 monomer is formed. And then the monomer NPR1 is directly targeted by the Pseudomonas Syringa effector AvrP to B, AvrP to B interacts with NPR1 monomer and ubiquitinates NPR1 monomer by SA dependent way mediated by its Cullin 3 ubiquitin E3 ligase ${ }^{[24-26]}$. The phosphorylation of monomer NPR1 at serine589 (s589) via protein kinase SnRK 2.8 is of great significance for the nuclear localization of NPR1. The phosphorylation of NPR1 at serine55 and serine59 (S55/59) promoted the binding of NPR1 to transcription factors such as WRKY. When the SA level increases with pathogen recognition, NPR1 undergoes a series of modifications, including dephosphorylation of S55/59, ubiquitination and phosphorylation of serine 11 and 15 (S11/15), transforming the correlation of NPR1 from WRKY transcription factor to TGAs (TGACGTCA cis element binding protein factor), regulating the target gene family of TGA transcription factors NIM1-interacting protein (NIMINs), thus stimulating expression of PR gene ${ }^{[26]}$ (Figure 1). Pathogen effectors can target these kinases, phosphatases or sumo binding enzymes, modify the important residues of NPR1, or destroy the interaction between NPR1 and related proteins, which may potentially interfere with NPR1 signal transduction. In addition, effectors may also affect the transcription level of NPR1 by targeting various transcription factors that regulate NPR1 expression ${ }^{[27]}$.

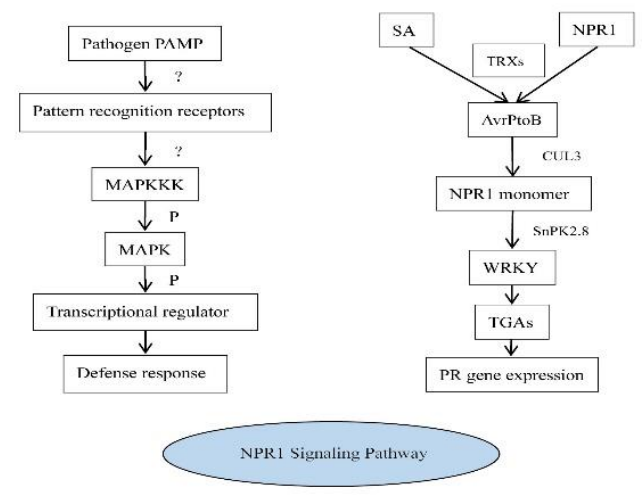

Figure 1. The Processes in the NPR1 Signaling Pathway

\subsection{NPR1 as a key regulator of SA signaling}

Most of the polypeptides encoded by PR gene are directly involved in the resistance of microorganisms. After being infected by pathogens or treated with SA, the accumulation of SA in plants can induce the change of redox state in plant cells, thus breaking the disulfide bond between the oligomer molecules of NPR1 to form NPR1 monomer and transfer to the nucleus. NPR1 is phosphorylated and then ubiquitinated after entering the nucleus. These processes are of great significance for the activation of SAR in plants. The accumulation of SA in plant can induce the change of redox state in plant cells, which makes NPR1 oligomer form NPR1 monomer and transfer to nucleus. NPR1 is phosphorylated and then ubiquitinated after entering the nucleus, which is of great significance for the activation of SAR in plants ${ }^{[24]}$. After entering the nucleus, any NPR1 monomer will be degraded gradually, but only phosphorylated NPR1 can activate $\mathrm{SAR}^{[28]}$. In addition to the regulation of PR gene expression with TGA transcription factors, NPR1 is also involved in the process of PR protein post-translational processing and transport to apoplast ${ }^{[29]}$. In addition, NPR1 is directly involved in the regulation of endogenous SA content. The activation of ics1 in SA synthesis pathway can promote the accumulation of SA, and then promote the transformation of NPR1 oligomer into NPR1 monomer and transport to the nucleus, which in turn inhibits the expression of ICS1 gene. When NPR1 cannot be transported to the nucleus, the continuous expression of ICS1 gene leads to excessive accumulation of SA, resulting in toxicity ${ }^{[30]}$.

\subsection{SA affects the interaction between NPR1, NPR3 and NPR4}

As the homologous gene of NPR1, both Npr3 and npr4 contain the BTB domain and an ankyrin repeat domain, which is a typical feature of Cul3 substrate adaptor protein. More importantly, although its sequence is similar to that of NPR1, npr3npr4 double mutants have the opposite 
phenotype with NPR1, showing enhanced disease resistance ${ }^{[24}$. NPR3 and NPR4 are receptors of the ubiquitinated E3 ligase cullin $3^{[28]}$, which act on the degradation of NPR1 before and after SA induction. SA affects the interaction between npr1-npr3 and npr1-npr4. NPR3 and NPR4 bind to SA with different affinity npr3npr4 double mutants have defects in SAR and ETI and are not sensitive to induced systemic acquired resistance. The interaction between NPR3, NPR4 and NPR1 is regulated by SA. SA inhibits the interaction between NPR4 and NPR1, but promotes the interaction between NPR3 and NPR1. NPR3 and NPR4 bind to SA with different affinity. When the amount of SA is very low, NPR4 and NPR1 combine to degrade NPR1, keeping NPR1 in the cell at a very low level; when the amount of SA is very high, Npr3 and NPR1 combine to degrade NPR1 in the cell nucleus; only when the content of SA is moderate, SA inhibits the interaction between NPR4 and NPR1, but it is not enough to promote the interaction between Npr3 and NPR1, at this time, NPR1 in the cell nucleus maintains at a high level, thus regulating downstream genes Expression. After the plant is infected by pathogenic bacteria, NPR3 and NPR4 act as receptors for SA, which determine cell death or survival by regulating the content of NPR1. The lower level of SA can inhibit the interaction between NPR4 and NPR1, but it is not enough to promote the interaction between NPR3 and NPR1. At this time, the accumulation of NPR1 protein can promote SA mediated resistance response and cell survival. Therefore, SA can regulate the degradation of NPR1 mediated by NPR3 and NPR4, and maintain it at a suitable level to determine the process of plant immune response ${ }^{[28]}$.

\section{Conclusion}

In Arabidopsis, rice ${ }^{[16]}$, apple ${ }^{[19]}$, tomato ${ }^{[31]}$, wheat ${ }^{[32]}$ and many other plants, it has been found that overexpression of NPR1 gene can improve plant disease resistance. Collectively, it is a common phenomenon that NPR1 regulates plant immune response in higher plants. NPR1 gene can induce SAR process to produce key regulatory components and participate in the activation of PR gene expression. After being infected by the pathogen, the SA level in the uninfected tissues increased. According to the types of plant resistance, the mechanism of NPR1 in plant resistance can be divided into the following three situations. the first belongs to basic resistance: in uninfected plant cells, the closed conformation of inactive NPR1 is mediated by direct interaction between BTB / POZ and trans activated (TA) domain. The second is that SA changed the conformation of NPR1 and induced homodimer of NPR3 and NPR4 in ETI pathway. Although SA promotes the interaction between npr1-npr3, it destroys the interaction between npr1-npr4 and npr1nimin2. The end is that in SAR, the intermediate concentration of SA can lead to the coexistence of inactive NPR1 and active NPR1 induced by binding SA ${ }^{[28]}$.

\section{Future considerations}

SA plays an important role in plant immune response. It can regulate the growth and development of plants through many ways. The mechanism of SA induced resistance is very important for crop protection. Although the combination of modern biochemical and genetic methods is conducive to the study of the synthesis and activation of SA in plants, SA receptor and the role of SA in biological and abiotic stress, it is still difficult to fully elucidate the mechanism of SA induced plant stress resistance, there are many problems still need to be solved: (1) this kind of signal network of SA is extremely fine, which is related to a variety of physiological and biochemical reactions, especially in plants Photosynthesis is extremely important. (2) the components and functions of the whole signal transduction pathway need to be fully defined. (3) even in different parts of the same plant, the pathways of SA signal transduction are not the same. Therefore, in different kinds of plants, this pathway remains to be studied.

As an important regulator, NPR1 plays an important role in SA signaling pathway. NPR3 and NPR4 are receptors in SA signaling pathway, which is a great progress in SA signaling research. NPR3 and NPR4 can bind to SA, but whether NPR1 binds directly to SA remains to be further studied. The binding of SA to NPR3 and NPR4 regulates the content of NPR1, and then regulates SA mediated resistance response and SAR establishment, but why NPR3 and NPR4 have different binding ability to SA. And SA participates in plant abiotic stress response process, but the biochemical mechanism of SA mediated abiotic stress response remains to be studied. At present, the mechanism and function of NPR2, NPR5 and NPR6 has not been reported.

Many studies have proved that SA, as an intracellular signal molecule, can participate in the biological and abiotic resistance of plants, especially in the defense of pathogenic microorganisms, which will cause the immune response triggered by PAMPs and effectors. In this process, SA will induce synthesis in chloroplasts. The signal transduction pathway of SAR induced by SA in plants is a complex network. With the rapid development of molecular biology technology, the research on NPR could make the whole pathway and induction mechanism clearer.

\section{Reference}

1. Boller T, Felix G. (2009) A renaissance of elicitors: perception of microbe-associated molecular patterns and danger signals by pattern-recognition receptors. Annu Rev Plant Biol, 60: 379-406

2. Macho AP, Zipfel C. (2014).Plant PRRs and the activation of innate immune signaling. Mol Cell, 54 (2): 263-272

3. Jones JD, Dangl JL. (2006) The plant immune system. Nature, 444 (7117): 323-329

4. Lewis JD, Guttman DS, Desveaux D. (2009) The targeting of plant cellular systems by injected type III effector proteins. Semin Cell Dev Biol, 20 (9): 10551063 
5. Mur LA, Kenton P, Llo yd AJ,et al. (2008) The hypersensitive response; the centenary is upon us but how much do we know? J Exp Bot, 59 (3): 501-520

6. Fu ZQ, Dong X. (2013) Systemic acquired resistance: turning local infection into global defense. Annu Rev Plant Biol, 64: 839-863

7. Piererse CMJ, Van Wees SCM, Van Pelt JA et al. (1998) A novel signaling pathway controlling induced systemic resistance in Arabidopsis. Plant Cell, 10:1571-1580

8. Mur LA, Kenton P, Atzorn R, et al. (2006) The outcomes of concentration-specific interactions betweensalicylate and jasmonate signal ing include synergy, antagonism, and oxidative stress leading to cell death. Plant Physiol, 140 (1): 249-262

9. Vlot AC, Dempsey DA, Kles sig DF. (2009) Salicylic acid, a multifaceted hormone to combat disease. Annu Rev Phytopathol, 47: 177-206

10. Endo J, Takahashi W, Ikegami T, et al. (2009) Induction of flowering by inducers of systemic acquired resistance in the Lemna plant. Biosci Biotechnol Biochem, 73 (1): 183-185.

11. Vicente M R S, Plasencia J. (2011) Salicylic acid beyond defence: its role in plant growth and development. J Exp Bot, 62 (10): 3321-3338

12. Blanco F, Salinas P, Cecchini N M, et al. (2009) Early genomic responses to salicylic acid in Arabidopsis. Plant Mol Biol, 70 (1-2): 79-102.

13. Wildermuth M C, Dewdney J, Wu G, et al. (2001) Isochorismate synthase is required to synthesize salicylic acid for plant defence. Nature, 414 (6863): 562-565

14. Cao H, Bowling SA, Gordon AS, et al. (1994) Characterization of an Arabidopsis mutant that is nonresponsive to inducers of systemic acquired resistance. Plant Cell, 6 (11): 1583-1592

15. Cao H, Glazebrook J, Clarke JD, et al. (1997) The Arabidopsis NPR1 gene that controls systemic acquired resistance encodes a novel protein containing ankyrin repeats. Cell, 88 (1): 57-63

16. Fitzgerald HA, Chern MS, Navarre R, et al. (2004) Overexpression of (At)NPR1 in rice leads to a BTHand environment-induced lesion-mimic/cell death phenotype. Mol Plant Microbe Interact, 17 (2): 140151

17. Lin WC, Lu CF, Wu JW, et al. (2004) Transgenic tomato plants expressing the Arabidops is NPR1 gene display enhanced resistance to a spectrum of fungal and bacterial diseases. Transgenic Res, 13 (6): 567581

18. Chern M, Fitzgerald HA, Canlas PE, et al. (2005) Overexpression of a rice NPR1 homolog leads to constitutive activation of defense response and hypersensitivity to light. Mol Plant Microbe Interact, 18 (6): 511-520

19. Malnoy M, Jin Q, Borejsza E E, et al. (2007) Overexpression of the apple MpNPR1 gene confers increased disease resistance in Malus $\times$ domestica. Mol Plant Microbe Interact, 20 (12): 1568-1580

20. Mukhtar, M.S. et al. (2016) Pathogen tactics to manipulate plant cell death. Curr. Biol. 26, R608R619

21. Liu G, Holub E B, Alonso J M, et al. (2005) An Arabidopsis NPR1-like gene, NPR4, is required for disease resistance [J]. The Plant Journal, 41(2):304318.

22. Zhang Y, Cheng Y T, Qu N, et al. (2006) Negative regulation of defense responses in Arabidopsis by two NPR1 paralogs [J]. Plant Journal, 48(5):647-656.

23. Delaney T P, Friedrich L, Ryals J A. (1995) Arabidopsis signal transduction mutant defective in chemically and biologically induced disease resistance. Proceedings of the National Academy of Sciences of the United States ofAmerica, 92(14): 6602-6606.

24. Spoel SH, Mou ZL, Tada Y, Spivey NW, et al. (2009) Proteasome-mediated turnover of the transcription coactivator NPR1 plays dual roles in regulating plant immunity. Cell, 137 (5): 860-872

25. Chen,H. et al. (2017) A bacterialtype III. Effector targetsthe master regulatorofsalicylicacid signaling, NPR1, tosubvert plant immunity. Cell Host Microbe 22, 777-788 e777

26. Pajerowska-Mukhtar, K.M. et al. (2013) Tell me more: roles of NPRs in plant immunity. Trends Plant Sci. 18, 402-411

27. Sun Y, Detchemendy T W, Pajerowsak K M,etal.(20 18)NPR1 in JazzSet with Pathogen Effectors. Trends in Plant Science, 23(6), 469-472

28. Fu ZQ, Yan S, Saleh A, et al (2012). NPR3 and NPR4 are receptors for the immune signal salicylic acid in plants. Nature, 486 (7402): 228-232

29. Wang D, Weaver N D, Kesarwani M, et al. (2005) Induc tion of protein secretory pathway is required for systemic acquired resistance. Science, 308 (5724): 1036-1040

30. Zhang X, Chen S, Mou Z (2010). Nuclear localization of NPR1 is required for regulation of salicylate tolerance, isochorismate synthase 1 expression and salicylate accumulation in Arabidopsis. J Plant Physiol, 167 (2): 144-148

31. Lin WC, Lu CF, Wu JW, et al. (2004) Transgenic tomato plants expressing the Arabidops is NPR1 gene display enhanced resistance to a spectrum of fungal and bacterial diseases. Transgenic Res, 13 (6): 567581

32. Makandar R, Essig JS, Schapaugh MA, et al. (2006) Genetically engineered resistance to Fusarium head blight in wheat by expression of Arabidopsis NPR1. Mol Plant-Microbe Interact, 19 (2): 123-129 